\title{
LA DIPLOMACIA ROMANA DURANTE EL PATRICIADO (509-367 A. DE J.C.)**
}

Emmanuel Mora Iglesias*

\begin{abstract}
RESUMEN
La diplomacia romana durante la era del patriciado (509-367 a.de J.C.) es el tema de este artículo. A través del análisis de fuentes textuales el autor se acerca, tanto de forma cuantitativa como cualitativa, a un tema reciente y poco estudiado.

Palabras clave: Historia Romana, Patriciado, Diplomacia, Historiografía Romana, Política Exterior Romana.
\end{abstract}

\begin{abstract}
The Roman Diplomacy during the years of the patrician political supremacy (509-367 B.C.) is the subject of this article. Through an analysis of text evidence the author presents a quantitative and qualitative approach to a rarely studied and quite recent subject.

Key Words: Roman History, Patriciate, History of Diplomacy, Roman Historiography, Roman Foreign Politics.
\end{abstract}

\begin{abstract}
Alla Romana
Para el lector es importante ubicarnos en que consistía la diplomacia en la Antigüedad. En la introducción de la muy reciente obra intitulada Diplomats and Diplomacy in the Ancient World escrita por Claude Eilers especifica el tipo de diplomacia existente en este período. En primer lugar las Ciudades-Estado o los imperios de este periodo no tenían, como el servicio diplomático moderno, oficinas que representasen sus intereses o que ayudasen a sus ciudadanos en el extranjero. No existía tampoco un Ministerio de Relaciones Exteriores ío ningún tipo de especialización solamente en diplomacia ${ }^{1}$. Como bien lo menciona el autor la diplomacia de esta época no era enfocada, como lo es la moderna,
\end{abstract}

a las relaciones entre Estados soberanos, sino que las relaciones se hacían entre comunidades con muy diferentes tipos de estatus y las que empleaban el mismo procedimiento, ritual y vocabulario entre ellas.

El procedimiento era el siguiente:

Thus legati $(\pi \rho \varepsilon \sigma \beta \varepsilon v \tau \alpha i / \pi \rho \varepsilon \sigma \beta \varepsilon 1 \sigma) \quad$ conduct legationes $(\pi \rho \varepsilon \sigma \beta \varepsilon v \tau \alpha l)$ to Rome (or elsewhere), where they approach a magistrate, who grants them access (senatum dare; $\sigma v \gamma \kappa \lambda \eta \tau o v \delta 1 \delta o v \alpha 1)$; they are heard with varying degrees of interest or approval, and sent on their way sometimes with official gift to cover their expenses (munus; $\zeta \varepsilon v 1 \alpha^{2}$ ).

Roma formó entonces una organización política pre-estatal con la consiguiente falta de una burocracia especializada en las funciones de gobierno $^{3}$. Los legados eran enviados a misiones

\footnotetext{
* Profesor, Escuela de Filología, Lingüística y Literatura, Universidad de Costa Rica.

** Artículo correspondiente a la Sección especial Luciana Sparisci in honórem (XXXVI/2/2012).

Recepción: 12/05/11 Aceptación: 28/06/11
} 
ad hoc para resolver situaciones precisas y determinadas ${ }^{4}$. Recordemos que por diplomacia entendemos el arte y la habilidad de comunicar e intercambiar información entre distintos países Estados o pre-Estados. La diplomacia es una forma de disuasión ${ }^{5}$. Al ser disuasoria los hombres con práctica y experiencia en la retórica, que es una forma disuasoria por excelencia, eran los indicados para este campo, esta experiencia se encontraba muchas veces en la práctica de la vida política de la ciudad. Como en Grecia los hombres con una experiencia en política y con una autoridad natural se encontraban en los consejos de su ciudad, en Roma en el Senado ${ }^{6}$.

\section{Ars Diplomatica}

En el año 1974 sale a la luz la obra de Sir Frank Adcock y D.J. Mosley intitulada Diplomacy in Ancient Greece ${ }^{7}$. En el prólogo de la obra se nos indica que era la intención del Prof. Adcock escribir no sólo un libro sobre diplomacia griega sino también romana ${ }^{8}$. Al parecer la obra sobre diplomacia romana nunca llegó a escribirse y la obra sobre diplomacia griega de este autor trata tan solo tangencialmente el tema y únicamente en relación con el contacto de Roma con el resto del mundo helenístico (a partir de 217 a.de J.C.). En 1999 hace su aparición la obra Kinship Diplomacy in the Ancient World 9 de Christopher Jones donde aborda el tema de relación de la diplomacia con los esquemas míticos de la Antigüedad. El capítulo 7 habla sobre la República romana y es vista desde este punto de vista. Finalmente en 2008 aparece la obra Diplomats and Diplomacy in the Roman World $^{10}$ que trata sobre aspectos muy concretos de la diplomacia romana luego de la invasión gala. Tenemos también los artículos publicados por F. Canali De Rossi "Le ambascerie dal mondo Greco a Roma in età reppublicana" que trata también una época histórica posterior ${ }^{11}$.

De acuerdo a las fuentes que hemos podido consultar aún no se ha escrito nada específicamente sobre la diplomacia en la época de la monarquía (753a. de J.C.-509 a. de J.C.) ni en la de los primeros tiempos de la República, salvo en obras en donde se la trata como parte del tema del expansionismo romano. Nuestra intención es hacer un primer estudio con las fuentes que disponemos que pueda extenderse en una obra de mayores proporciones y que logre llenar un vacío existente en el tema.

\section{Ab Antiquo}

La Historia Romana tiene la particularidad, mencionada por Tim Cornell, de usar como como fuentes primarias, fuentes que en otros tipos de historia serían consideradas como secundarias. Son fuentes escritas por historiadores los que han seleccionado e interpretado ya los hechos de acuerdo a su subjetividad $^{12}$. Las fuentes que disponemos para este tema son de carácter literario. En primer lugar tenemos al historiador Tito Livio (59-17 a.de J.C.). En su obra $A b$ Urbe Condita (desde la Fundación de la Ciudad), nos narra la historia de la ciudad desde la fundación de la República hasta su época, el Siglo de Augusto. El propósito de su historia era moralizante, poner a los romanos al frente de la grandeza moral de su historia frente a los nuevos tiempos que corrían. Obviamente este prejuicio del autor afecta la objetividad de su obra en donde los hechos de romanos ilustres son dados como exempla a las nuevas generaciones. Su obra la componían 142 libros (sólo 35 sobreviven) de los cuales los cinco primeros cubren los orígenes de la ciudad hasta el saqueo de Roma. Livio, como sus contemporáneos, nunca cita sus fuentes, salvo en caso de duda o de conflicto. Al parecer empleó material de los analistas romanos Licinio Macer (muere en 66 a.de J.C. y escribe una historia de Roma en 16 tomos), Aelio Tubero (jurista y cónsul suff.118 a.de J.C.), Fabio Pictor (ca.254 a.de J.C.-¿?, escribe crónicas sobre la historia romana) y Calpurnio Piso (Pretor 211 a.de J.C.). Estos autores viven sin embargo (en el caso de Piso y de Pictor) unos 300 años luego de los hechos que narraron. Sabemos que los annales maximi, fuente histórica de primera mano sobre la época temprana romana, fueron destruidos tras el saqueo celta de Roma (ca.390 a.de J.C.). También sabemos que las familias aristocráticas romanas tenían sus archivos privados, que en 
caso de haber preservado los hechos de los primeros tiempos de los romanos, debieron servir de fuente a los historiadores posteriores. La tradición oral también debió de haber tenido un papel nada despreciable. Así mismo las fuentes historiográficas griegas registran los hechos de Roma desde el saqueo de los galos de la ciudad. Los dos principales historiadores griegos de esta época son Jéronimo de Cardia (muere en 250 a.de J.C.) y Timeo de Tauromenio (356-260 a.de J.C.). En qué medida estos historiadores fueron usados por los analistas y subsecuentemente por Livio no nos es posible en este momento saberlo. Livio en una parte de su obra hace una digresión en la que discute precisamente el objeto de su estudio y las dificultades para el mismo. Nos dice que "El objeto de estudio está envuelto en oscuridad, en parte por su gran antigüedad, ya que los objetos remotos son costosamente visibles a través de la vastedad de la distancia temporal y en parte debido al hecho de que los registros escritos, de los cuales existían pocas y valederas listas de eventos, y aún lo poco que existía en los comentaros pontificales y en los archivos privados y públicos pereció en el la destrucción de la ciudad ${ }^{13}$.

Nuestra otra fuente es Dionisio de Halicarnaso. Este historiador vivió en Roma desde aproximadamente los 30 a.de J.C. Hacia el 7 a.de J.C. comienza la publicación de su

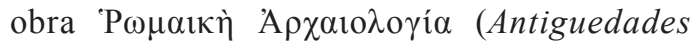
Romanas) en la que trata reconciliar al público griego con el dominio romano. Al ser esta obra un panegírico tiene un estilo retórico (el mismo Dionisio enseñó retórica mientras estudiaba latín en Roma) y muchas de sus partes transmiten además discursos cuya veracidad es discutible. Hace uso de fuentes de los analistas, historiadores y algunos documentos públicos que disponía en su época. De sus veinte libros de historia sobreviven los diez primeros y es un excelente complemento a la historia de Tito Livio.

En Plutarco (50 d .de J. C.-120 d .de J. C.) tenemos también importantes referencias. Este autor, de origen griego nacido en Queronea (sitio de la famosa batalla que vió nacer el genio militar de Alejandro Magno). Este escritor estuvo en Atenas, visita Egipto e Italia y es maestro en Roma. Plutarco es sacerdote en Delfos en los ultimos 30 años de su vida. El mismo Plutarco fue magistrado de Queronea y sirvió como embajador en varias ocasiones representando su ciudad natal. Escribió unos 78 tratados de distintos temas y unas 50 biografías, de las que sobrevien tan solo dos de los césares y 23 pares de las vidas paralelas. Las dos vidas paralelas que empleamos son las de Cayo Marco Coriolano (s.V a.de J.C.) y la de Marco Furio Camilo (446-365 a.de J.C.).

Algún detalle hemos encontrado en Dio Cassio (consul suff. 205 d.C), basado en Livio o en sus fuentes.

\section{La Fundación de la República romana y el inicio del Patriciado}

En 509 Tarquino el Soberbio, último rey de Roma fue expulsado y la monarquía abolida. Se establece así una república aristocrática en la que el poder regio es sustituido por dos cónsules electos anualmente y revestidos con imperium ${ }^{14}$. Dos conflictos marcarán la historia romana de este período, uno externo, contra los pueblos vecinos de Roma, y otro interno, de carácter social. Hay que recordar que los romanos estaban divididos en dos clases; patricios y plebeyos. Estos últimos eran desfavorecidos en materia económica y no tenían acceso a las magistraturas en Roma. Como resultado de esta lucha de los órdenes los plebeyos establecen con el tiempo sus propias magistraturas (el tribunado y el edilato) así también como su propia Asamblea (Concilium Plebis).

Distintas leyes e instituciones manifestaron el cambio social en Roma. Así tenemos la Lex Publilia Voleronis (471), la aparición de los Decemviri y la codificación y publicación de la Ley de las XII Tablas, las Leyes Valerio-Horacias (449), La Lex Canuleia (445), la rogaciones Licionio- Sextias (367). Desde 455 a 376 existen los Tribuni Militum Consulari Potestate que reemplazan temporalmente a los cónsules, magistratura que ya estaba abierta a los plebeyos, y posteriormente las funciones de 
estos tribunos pasarán a los cénsores cuando se crea esta magistratura en 351 y a los pretores en 337 .

Todas estas luchas sociales tenían un origen económico tras la carencia de suficiente alimento, tierra y al tenerse también leyes severas contra los deudores. Estos problemas parecieron aliviarse con la conquista de Italia y la redistribución de la tierra.

\section{Las primeras embajadas de una ciudad-estado constituida en república}

Según la bien conocida leyenda Roma fue fundada en 753 a.de J.C. por dos hermanos, Rómulo y Remo, quienes inician un sistema de gobierno monárquico que se extenderá hacia el 509 a.de.J.C. ${ }^{15}$ fecha en la cual el último soberano, Tarquino, apodado el Soberbio y de origen etrusco, fue expulsado de la ciudad.

Roma al abolir la monarquía mantiene la constitución de su ciudad pero se convierte en una república aristocrática ${ }^{16}$. Esta tiene como vecinos a diversos pueblos. Al Norte estaban los etruscos, al Nordeste los sabinos, al Este los ecuos y al Sudeste los volscos ${ }^{17}$. Los etruscos, llamados también tirrenos, tenían un origen desconocido y alcanzaron la cima de su poder en la península itálica hacia 500 a.de J.C. Los sabinos por su parte, también de origen desconocido, es probable que hablasen osco como lengua (emparentado con el latín y consecuentemente de origen indoeuropeo). Estos habitaban las faldas de los Apeninos. Los volscos en el siglo VI a.C. llegan a la Italia Central y poseían varias ciudades tales como Sora, Arpinum, Anxur, Circeii, Privernum Atina, Velitrae. Hablaban una lengua parecida al umbrio (y consecuentemente al osco). Finalmente los ecuos (aequi) eran una tribu itálica primitiva y hablaban una lengua parecida al osco. Se expanden hacia el Lacio ca.del 500 a.de J.C. Se establecen en los montes albanos.

La llanura del Lacio era probablemente deseada por todos ellos ${ }^{18}$. Roma había perdido su preponderancia en el Lacio a raíz del cambio de régimen ${ }^{19}$. La primer embajada que recibe
Roma durante su vida republicana (509 a.de J.C.) es precisamente la de los legados de la familia real que llegan con la intención de recuperar los bienes del rey destronado. Al llegar a Roma son admitidos en audiencia en el Senado y según Dionisio de Halicarnaso pronuncian un discurso en defensa del rey destituido ${ }^{20}$. Sin embargo, secretamente intrigaban para la restauración del antiguo régimen ${ }^{21}$. Esta conspiración fue descubierta por un esclavo que la descubre al estar estos cenando en la casa de los Vitelios (una familia patricia romana). Los legados fueron enviados a prisión y se nos dice que no existía certeza del trato que debía aplicarseles lo que obviamente indica una inexperiencia en los procederes en relaciones exteriores de parte de los romanos. Al final es interesante notar que el derecho de gentes (Ius Gentium) es al final tomado en cuenta por su condición de legados y entonces no se les somete a todo el peso de la ley a pesar de habérseles descubierto hechos que amenazaban el nuevo status quo 22 . El peligro de que los Tarquinos, con ayuda del rey etrusco Lars Porsena de la poderosa ciudad de Clusium, recuperen la ciudad, motiva que los romanos despachen la primer embajada en su vida republicana de la que tenemos registros. Los legados fueron enviados a las Ciudades-Estado de Cumas (una colonia griega cerca de Nápoles fundada por Calcis hacia el 750 a. de J.C.) y de los Volscos para adquirir grano (annonae) con el objeto de que el Senado lo distribuyese entre la plebe y así asegurarse su apoyo ante el peligro exterior inminente ${ }^{23}$. De acuerdo con Dionisio de Halicarnaso las embajadas enviadas fueron más bien una a los latinos-y no a los volscos(solicitando su ayuda ante la inminente guerra con Clusium) y efectivamente, a Cumas, con el propósito de adquirir grano. Los latinos se niegan hábilmente a brindar esta ayuda ya que afirmaban que poseían tratados de alianza tanto con Clusium como con Roma y que no podían traicionar a ninguno de los $\operatorname{dos}^{24}$.

Una embajada amenazante del mismo Lars Porsena es enviada antes del comienzo de las hostilidades entre Roma y Clusium ${ }^{25}$.

Los legados que luego tenemos noticia son enviados por las tropas de esta misma 
ciudad de Clusium que asedian Roma con el fin de levantar el sitio. Estos presentan la propuesta para la restauración de la casa real, probablemente con el fin de quedar bien a los ojos del destituido Tarquino, pero no con la intención real de que los romanos la aceptasen. Por otro lado las demandas para la restitución de territorio a la ciudad de Veyes son aceptadas y la paz se acuerda ${ }^{26}$.

En 507 a.de J.C. Roma despacha a los Sabinos unos legados con el fin de escuchar sus explicaciones con relación a una invasión de territorio ${ }^{27}$.

En el año 506 a.de J.C. Lars Porsena manda una embajada de nuevo a Roma con el propósito de convencer a los romanos a restaurar a Tarquino y acto inmediato los romanos responden con una misión, encabezada por el más ilustre de los senadores (cuyo nombre no nos es proporcionado) los cuales tienen un éxito rotundo: disuaden a Porsena a no intentar más restaurar a Tarquino (al cual no se le dará más asilo y deberá marcharse con su hijo Octavio a Túsculo), a devolver los rehenes y restaura también el territorio veyente que había tomado con el tratado ${ }^{28}$. Todos estos hechos debieron ocurrir poco después de la abolición de la monarquía (509 a.de J.C.)

En 507 el Senado envía una embajada a los sabinos con el propósito de pedir cuentas sobre la invasión de parte del territorio romano y poco tiempo después estalla la guerra ${ }^{29}$. Cinco años después la guerra termina con la victoria de los romanos. Los sabinos dirigen una embajada a Roma para concretar un tratado de paz con sus vencedores ${ }^{30}$.

En 501 Marcus Valerius, en nombre del Senado, se encamina a las villas latinas cercanas a Roma (las fuentes nos mencionan tan solo Ferente) para exigirles no hacer problemas con Roma y detener las bandas de rapiña instaladas en estas ciudades ${ }^{31}$. El legado romano descubre que las ciudades latinas están preparando la guerra contra Roma.

En 500 a.de J.C. Roma remite una embajada a la ciudad etrusca de Fidenas. Esta misión estuvo en esa ciudad a punto de ser tratada como enemiga de parte de las tropas enviadas por los tarquinos y también de parte de los habitantes de la ciudad. Al final no se les admite ni se les da audiencia $^{32}$. Por su parte Fidenas expide después a Roma una embajada exigiendo restablecer el antiguo régimen y dar una amnistía general para los exiliados ${ }^{33}$. Algunos de los emisarios que llegan a Roma tienen una fuerte suma de dinero con el propósito de sobornar a los más pobres y atraerlos a la causa de los Tarquinos ${ }^{34}$. Estos embajadores son expulsados de la ciudad luego de haberse descubierto su trama ${ }^{35}$. Ya en guerra Roma recibe una embajada proveniente de las 30 ciudades latinas coaligadas con el propósito de solicitar la paz y compuestas por sus principales ciudadanos. La ciudades eran Ardea, Aricia, Boville, Bubente, Cornea, Circea, Coriola, Cortante, Laurente, Launia, Lavinion, Labica, Nomenta, Morea, Preneste, Pede, Corccotula, Satrica, Escapra, Sete, Tellena, Tibur, Tusculum, Toleria, Tricina y Velitra. Esta es rechazada ${ }^{36}$, Roma por su parte remite embajadas a otras ciudades vecinas (Hernicia, que rechaza la oferta, y Rotula, que la acepta, son mencionadas) con el fin de asegurarse su alianza ${ }^{37}$. En 498 Roma envía embajadas de forma secreta a otras ciudades del Lacio con el fin de terminar la guerra y se consigue una tregua ${ }^{38}$. Los volscos envían también una embajada, al campamento romano en la batalla del lago Regilo, con el propósito de entablar relaciones amistosas (pero de acuerdo a Dionisio de Halicarnaso por razones de espionaje ${ }^{39}$ ). Unas cartas interceptadas hacen patente el doble juego de los volscos ${ }^{40}$.

La guerra contra los latinos se decidirá en esta batalla (Regilo) en 496 y que dará como resultado una nueva alianza de los latinos para con los romanos en 494. Este es el primer tratado internacional de Roma como república y el cónsul Espurio Casio (Spurius Cassius) es el que lo lleva a cabo ${ }^{41}$. La guerra volverá a estallar hasta 486 , esta vez contra los ecuos y los volscos, y durará, de forma intermitente, por cincuenta años.

Sabemos de una embajada que los volscos envían a los latinos con el fin de que estos reanudasen la guerra contra Roma; sin embargo, estas fueron entregadas por los latinos 
a los romanos. Se descubre que los volscos y los hernicios estaban planeando la guerra contra Roma. Los latinos envían por su parte una embajada a Roma con una corona de oro a Júpiter Capitolino en Roma y regresan con seis mil antiguos prisioneros latinos liberados por Roma. Se consolida así la alianza latino-romana ${ }^{42}$.

En 494 a.de J.C. los romanos, ante la escasez de grano, envían una embajada a los tirrenos (o sea a los etruscos), a los campanos y a la llanura Pomptina (en otras palabras a los volscos) con el fin de adquirir todo el grano que pudieran y manda a Sicilia, en 490, a Publio Valerio y a Lucio Geganio ${ }^{43}$. Los embajadores enviados a la llanura Pomptina fueron condenados como espías y estuvieron a punto de morir a manos de los volscos en tanto que los llegados a Cumas fueron objeto de ataque de los exiliados romanos de antiguo régimen que estaban en la Ciudad ya que estos pidieron al tirano que se les diera la muerte o se les retuviese como rehenes ${ }^{44}$. Tan solo los embajadores enviados a Tirreno (Etruria) cumplieron con su cometido ${ }^{45}$. En este mismo año la Plebe se retira en la primera Secessio al Monte Sacro (Mons Sacer) y la legación enviada por el Senado para hablar con ellos es encabezada por el antiguo cónsul Menenius Agrippa ${ }^{46}$.

Plutarco reporta una embajada de Velitrae (antigua ciudad volsca y donde muchos años después habría de nacer el primer emperador romano, Augusto) que llega a Roma solicitando nuevos habitantes para repoblarla ${ }^{47}$.

En 491 el Senado manda legados a los anciates quienes se niegan a seguir las directrices romanas ${ }^{48}$.

Los latinos expiden una embajada a Roma para solicitar ayuda contra los ecuos que habían invadido su frontera ${ }^{49}$.

Con respecto a la reanudación de la guerra intermitente contra los ecuos y los volscos (496446 a.C) en 489a.de J.C. los primeros despachan una embajada a Roma con el propósito de poner fin a las querellas a cambio de la recuperación de las ciudades que los romanos les habían quitado ${ }^{50}$. Luego, se menciona que el cónsul
Quinto Fabio destina una embajada a los volscos (485 a.de J.C.) con el fin de que aceptasen la paz.

En 489 a.de J.C. una embajada de la Liga Latina es despachada a Roma con el propósito de pedir ayuda militar, la cual les es denegada en virtud de los tratados ${ }^{51}$. También, los volscos remiten una embajada con el propósito de obtener la rendición de Roma tras una serie de campañas victoriosas de conquista frente a los aliados latinos de esta ciudad y que comandaba el tristemente célebre Coriolano ${ }^{52}$. Una nueva embajada, integrada por 10 ex-cónsules, es enviada de regreso con el propósito de que los volscos levantaron el asedio con que mantenían a Roma; sus negociaciones fueron infructíferas. Poco después Roma acuerda el envío de otra embajada "mayor y más honrosa" compuesta ahora de los augures, pontífices y ministros del culto más preclaros (tanto por sus ancestros como por sus hechos) y que llevarían consigo símbolos y hábitos sagrados ${ }^{53}$. Sus gestiones fracasan también frente a la negativa volsca ${ }^{54}$. El asedio se levanta por una embajada, no oficial, de mujeres encabezada por una patricia romana, Valeria, y otras mujeres romanas que logran convencer al general enemigo (Coriolano) para que termine el asedio ${ }^{55}$.

En 487 a.de J.C. Roma remite una embajada a los hernicios con el fin de pedirles reparaciones por las incursiones a las que Roma se vió sometida durante la expedición de los volscos y ecuos ${ }^{56}$.

En 482 a.de J.C. una misión de Roma llega a la ciudad etrusca de Veyes con el propósito de procurarse satisfacciones debido a expediciones de rapiña al territorio romano de parte de los veyentes. Tras la respuesta de Veyes, que clamaba su inocencia en este caso, los enviados encuentran pruebas del botín romano capturado por Veyes declaran la guerra ${ }^{57}$.

En 481 a.de J.C. llegan a Roma embajadores de los latinos pidiendo asistencia militar contra los ecuos, petición esta que fue satisfecha ${ }^{58}$.

En 478 a.de J.C. los latinos envían una embajada clamando por ayuda ante la invasión de los tirrenos (etruscos ${ }^{59}$ ). Ante el sitio de la ciudad y su inminente derrota los veyentes 
se presentan ante el cónsul con una misión integrada por los más ancianos con ramos de suplicantes para hacer la paz. Sus súplicas culminan en un armisticio $^{60}$.

En 477 a.de J.C. los veyentes expiden de nuevo una embajada a los romanos presentando una queja por la construcción de la fortaleza de Cremera y declarar la guerra en caso de la negativa de los romanos ${ }^{61}$. Ya declarada de nuevo la guerra, y con el revés de las armas, se repiten de nuevo los hechos del año anterior. Los veyentes salen al encuentro del cónsul con ramos de suplicantes y solicitando la conclusión de la guerra. El cónsul exige a Veyes el dinero para la paga de una año del ejército, alimento para dos meses y el envío de una embajada veyente con destino a Roma para hacer las formalidades del caso ${ }^{62}$.

En 466 a.de J.C. los latinos claman ayuda militar ante el Senado ante la violación de los acuerdos de parte de los ecuos y la devastación a que estaban siendo sometiendo sus territorios. Los romanos eligen tres embajadores, a cuya cabeza estaba Quinto Fabio, y los remite a los ecuos con el propósito de informarse de la responsabilidad del Estado con respecto a los hechos que se les atribuían. Los enviados encuentran preparativos para la guerra en la ciudad, comunican al Senado lo visto y Roma declara la guerra ${ }^{63}$.

En 451 a. C. se menciona la primera embajada romana durante este período fuera de la península itálica (y de Sicilia). Se realizó durante el período de los decenviros (451450 a.de J.C.). Tres comisionados, que sabían sobre leyes extranjeras, llegan a Atenas con el propósito de compilar nuevas leyes ${ }^{64}$. Los embajadores fueron Espurio Postumio, Servio Sulpicio y Aulo Manlio ${ }^{65}$.

En 449 a.de J.C. tiene lugar otra secesión de la plebe. Se menciona una embajada de carácter interno enviado por el Senado a los Plebeyos instalados en el Monte Aventino. La misión les pregunta con que autoridad se habían escindido. Los plebeyos exigen que se les envíen nuevos legados, en esta ocasión a Lucio Valerio y Marco Horacio a quienes les darían una respuesta en debida forma ${ }^{66}$.
Alrededor de 444 a.de J.C. se había renovado un antiguo tratado con la ciudadestado de Ardea y se recibe la embajada de la misma solicitando ayuda a Roma que a causa de las luchas internas se encontraba en la ruina. Los nobles eran los que acudían a Roma en tanto que la plebe se inclinaba por la ayuda de los volscos ${ }^{67}$.

Se nos menciona el nombre de una embajada que es enviada a Veyes con el propósito de investigar sobre el cambio de política en la ciudad la cual es asesinada por orden del mismo rey. A su vez, se explicitan los nombres de los legados: C. Fulcinius, Cloelius Tullius, Sp.Antius y L.Roscius. En la fuente queda claro que este hecho no era normal sino una violación a la ley de las naciones (Lex Gentium). En Roma este hecho tuvo consecuencias inmediatas; las estatuas de los enviados asesinados fueron expuestas en las Rostras, sitio de gran importancia en el Foro y desde donde los oradores llevaban a cabo sus arengas.

Ante la posibilidad del inicio de una nueva guerra con Veyes el Senado romano manda una embajada, la cual es recibida de mala forma y la cual es amenazada con la muerte en caso de no retirarse rápidamente. En consecuencia de esto el Senado emplaza a los tribunos para presentar la moción de guerra ante las centurias lo antes posible ${ }^{68}$.

Marco Furio Camilo, logra derrotar a la ciudad etrusca de Veyes que había sostenido un asedio por diez años(396 a.de J.C.). Los etruscos ya en esta época estaban resistiendo los embates de los celtas los cuales desde el Norte se habían movilizado con el propósito de saqueo y conquista. Antes de la conquista de Veyes una embajada compuesta por Licinio Cosso (Licinius Cossus), Valerio Potito (Valerius Potitus) y Fabio Ambusto (Fabius Ambustus) es enviada a Delfos con el propósito de indagar los portentos y consultar el Oráculo de Delfos. La respuesta de Apolo no se hace esperar, vuelven los legados con algunas instrucciones y luego la ciudad de cae. Una nueva embajada integrada por tres legados es despachada en agradecimiento a Delfos con un lavamanos de oro (8 talentos de oro $\left.{ }^{69}\right)$. 
Una embajada de los faliscos llega a Roma con el objeto de someterse a Roma. Esta visita culmina en un tratado de alianza ${ }^{70}$.

En 390 una horda de senones (tribus celtas) aparecen ante los muros de Clusium. Esta ciudad manda legados a Roma con el fin de asistencia militar (a pesar de que no tenía tratados de alianza o de amistad). No obtienen esta asistencia pero Roma manda una embajada a dialogar con los celtas. Esta estaba compuesta por los tres hijos de M.Fabius Ambustus y amenazan a los celtas a no hacer la guerra a ningún amigo o aliado de Roma (suponemos que los romanos habrían dado un reconocimiento tácito de Clusium como una ciudad-estado amiga ${ }^{71}$ ). Los celtas ponen como condición a la paz la cesión de parte de Clusium de una parte de su territorio y que la respuesta de los de esta ciudad sea dada en presencia, aparte de los celtas, de los enviados romanos. Los ánimos se agitan y se da una batalla entre los etruscos junto a los enviados romanos por un lado y los celtas por el otro. El enviado Q. Fabius muere en la lucha ${ }^{72}$.

Los celtas mandan una embajada a Roma para protestar contra la violación del Lex Gentium de parte de los legados romanos. El Senado reconoce el error de los Fabii y los reprende. Sin embargo, debido al poder e influencia de esta misma familia, estos mismos Fabios son elegidos como tribunos consulares al año siguiente, cosa que los celtas interpretan como un acto hostil y los legados celtas, amenazando con la guerra, regresan a su campamento y se encaminan a una guerra que conducirá a la derrota romana en las márgenes del río Alia y la conquista temporal de la ciudad por los recién llegados ${ }^{73}$.

\section{Todos los caminos conducen a Roma}

Si hacemos un análisis comparativo del origen y de destino de las embajadas desde y hacia Roma obtendremos, con base en los datos proporcionados tanto por Tito Livio como por Dionisio de Halicarnaso, que el mayor peso de las relaciones exteriores de Roma eran con la Liga Latina. Un 28 por ciento de las misiones que
Roma recibió en este período venían de ahí y un 22 por ciento de las misiones enviadas por Roma hacia el exterior iban hacia allí. En segundo lugar tenemos a la ciudad etrusca de Clusium donde también un 28 por ciento de las legaciones de visita en Roma procedían de esa ciudad en tanto que tan sólo un 1 por ciento de las misiones romanas tenían a Clusium como destino. Es interesante el porcentaje correspondiente a los ecuos y a los volscos, pueblos con los que Roma tuvo una larga guerra de carácter intermitente que duró cincuenta años (496-446 a.de J.C.). El intercambio de embajadas entre Roma con los ecuos y los volscos fue mínimo alcanzando si acaso un 10 por ciento del total. Es destacable también el hecho de que la mayor parte de las relaciones diplomáticas de Roma en este período estaban confinadas al Lacio y a la Etruria, con contadas relaciones con la Magna Grecia (Cumas y Sicilia) las que eran no tenían objetivos políticos-militares sino enviadas por motivos puramente económicos (la compra de grano). La única embajada fuera del ámbito de la península Italiana y Sicilia, fue la misión romana a Atenas en 451 con un propósito legal.

\section{Corpus Diplomaticum}

Las características básicas de la diplomacia romana de hace 2500 años se han conservado en la diplomacia de hoy. La diplomacia sigue siendo un arte de persuasión y en los que se siguen usando formas retóricas, tanto verbales como visuales con este fin. Sigue siendo el medio para llevar a cabo la política exterior de una entidad política. Los legados romanos (y de la Antigüedad) cumplían las mismas funciones de los diplomáticos modernos; transmitían la información que se les encomendaba entregar, escuchaban y promovían, en su contacto con los agentes externos, los intereses de su lugar de origen, y ayudaban en la construcción de una red externa de amigos y de aliados. Tanto en la Antigüedad como actualmente, su integridad personal estaba revestida de una inviolabilidad sacrosanta basada, en principio, en el Ius Gentium. 


\section{Nota sobre las fuentes de los gráficos}

La fuente de mayor valor para recabar estos datos ha sido Dionisio de Halicarnaso, el cual nos transmite una mayor cantidad de datos sobre el número y el tipo de las misiones romanas, sin embargo Livio ha sido un muy buen complemento ya que proporciona datos que no están contenidos en Dionisio. Los datos que hemos obtenido de acuerdo a las fuentes disponibles las cuales seguramente no han transmitido toda la información. Empero, creemos que con las fuentes disponibles ha sido posible trazar una tendencia general de las relaciones diplomáticas de Roma para un período en el que la expansión de esta emergente república estaba en su primera fase.

\section{CUADRO I}

Embajadas de y hacia Roma durante el Patriciado.

Según Tito Livio (59-17 a.de J.C.)

\begin{tabular}{cllll}
\hline Año & \multicolumn{1}{c}{ Origen } & Destino & \multicolumn{1}{c}{ Fuente } & Composición \\
\hline 509 & $\begin{array}{l}\text { Gens real (hab. } \\
\text { Clusium) }\end{array}$ & Roma & Liv. II.4. \\
& & Dion.Hal.V.1.7. &
\end{tabular}

\begin{tabular}{llll}
\hline 509 & Roma & Volscos y Cumas & Liv.II.9. \\
\hline & Clusium & Roma & Liv.II.13. \\
\hline 506 & Clusium & Roma & Liv. II.1
\end{tabular}

\begin{tabular}{|c|c|c|c|}
\hline ¿496? & Liga Latina & Roma & Liv. II.22. \\
\hline & Aricia & Roma & Liv. II.26. \\
\hline 465 & Roma & Ecuos & Liv. \\
\hline
\end{tabular}

\begin{tabular}{|c|c|c|c|c|}
\hline 451 & Roma & Atenas & Liv. III.33 & 3 legados \\
\hline 449 & Senado & Monte Aventino & Liv. III.50. & 3 legados \\
\hline ¿444? & Ardea & Roma & Liv. IV.9. & $\begin{array}{l}\text { C. Fulcinius, Cloelius Tullus, } \\
\text { Sp. Antius, L.Roscius }\end{array}$ \\
\hline
\end{tabular}

\begin{tabular}{lllllll}
\hline & Roma & Territorio Hernicio & Liv. & Tribunos Consulares \\
\hline & Roma & Veyes & Liv. IV.58 & & \\
& & & & \\
\hline 390 & Clusium & Roma & Liv.V.33. & & \\
\hline 390 & Roma & Campamento celta & Liv. 5.35. & $\begin{array}{l}3 \text { hijos de } \\
\text { Ambustus. }\end{array}$
\end{tabular}

\begin{tabular}{llll}
\hline 390 & Camp.celta & Roma & Liv. \\
\hline
\end{tabular}




\section{GRÁFICO I.}

Procedencia de las embajadas con Roma como destino (509-367 a.de J.C.). Fuente: T.Livio.
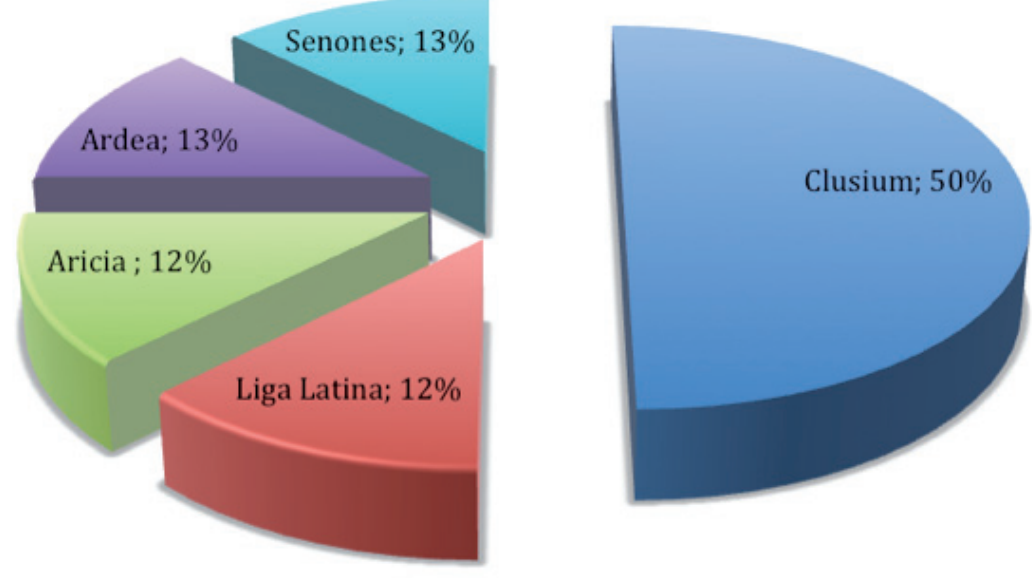

GRÁFICO II.

Procedencia de misiones a Roma (509-367 a.de J.C.) según Dionisio de Halicarnaso
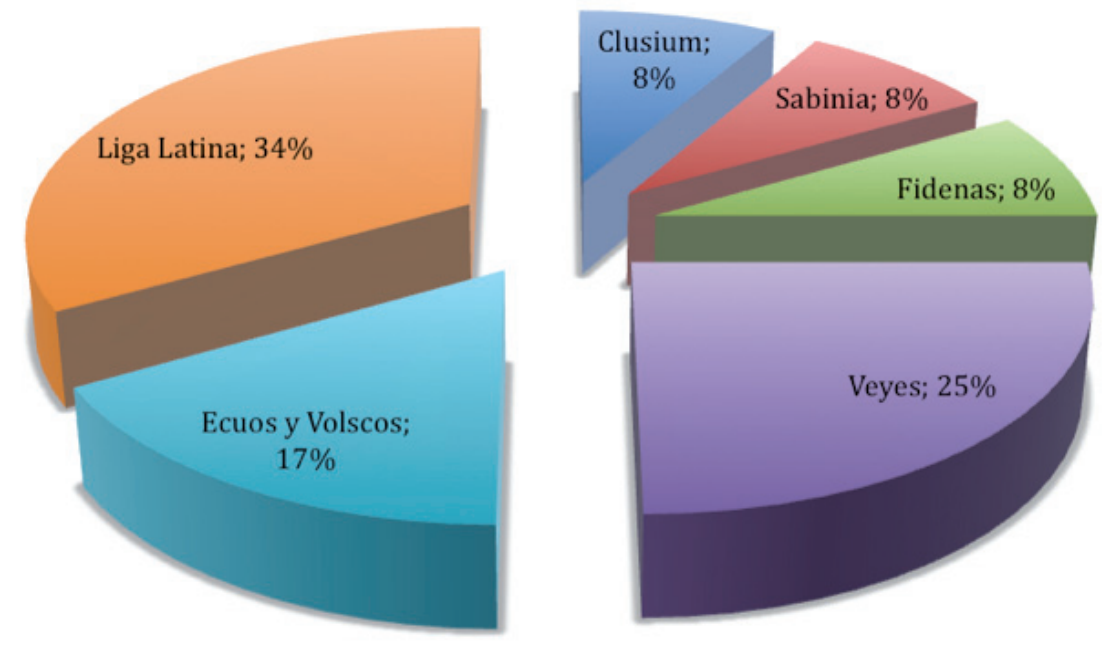
GRÁFICO III.

Origen de las misiones con destino a Roma (509-367 a.de J.C.).

Fuente: T. Livio, Dionisio de Halicarnaso.

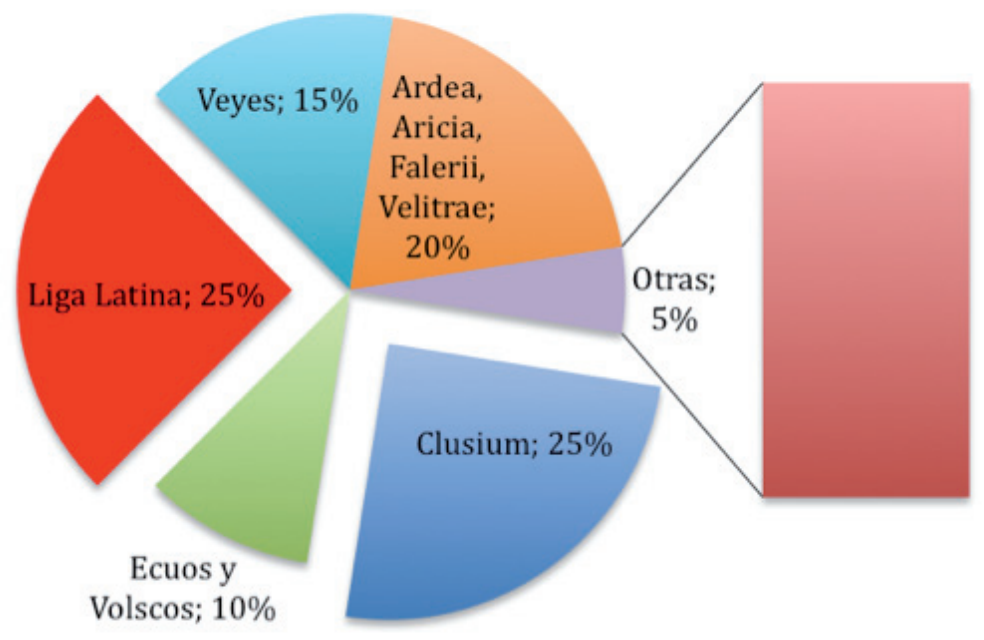

GRÁFICO IV

Destinos de las misiones romanas al exterior (507-367 a.de J.C.)

Fuentes: T.Livio, Dionisio de Halicarnaso.

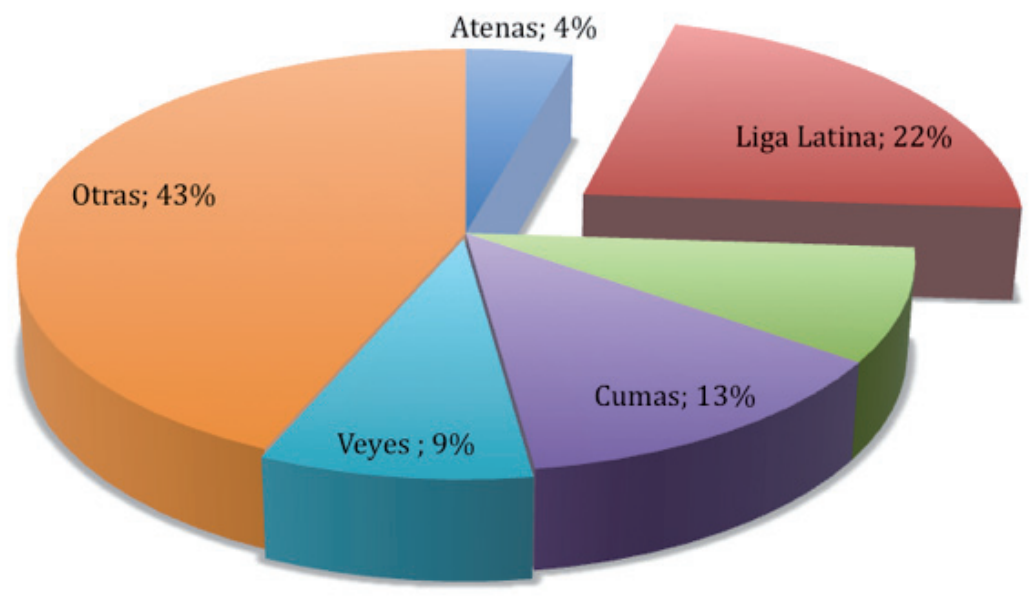


CUADRO II

Embajadas de y hacia Roma durante el Patriciado. Según Dionisio de Halicarnaso (ca.60-7 a.de J.C.)

\begin{tabular}{|c|c|c|c|}
\hline Año & Origen y Destino & Fuente & Composición \\
\hline 509 & Gens Real (Clusium)- & Dion.Hal.V.1-7. & ¿? \\
\hline \multicolumn{4}{|l|}{ Roma } \\
\hline & Clusium- Roma & Dion. Hal.V.4-1. & ¿? \\
\hline & $\begin{array}{l}\text { Roma- Liga Latina, Cumas } \\
\text { y ciudades de los Pomecios }\end{array}$ & Dion. Hal.V.4.14. & ¿? \\
\hline 507 & Roma- Sabinos & Dion. Hal.V.6.3. & ¿? \\
\hline 502 & Sabinos-Roma & Dion.Hal. V.9.1. & ¿? \\
\hline 501 & Roma-Ciudades latinas & Dion.Hal. V.10.2. & Marcus Valerius \\
\hline \multicolumn{4}{|l|}{ Ferente } \\
\hline \multirow[t]{4}{*}{500} & Roma- Fidenas & Dion. Hal.V.11.1. & ¿? \\
\hline & Fidenas-Roma & Dion.Hal.V.11.4. & ¿? \\
\hline & Liga Latina-Roma & Dion.Hal.V.11.8. & ¿? \\
\hline & Roma- Liga Latina & Dion.Hal.V.13.6-7. & ¿? \\
\hline 498 & Roma- Liga Latina & Dion.Hal.V.10.14. & ¿? \\
\hline 496 & Volscos-Roma & Dion.Hal.V.14.14. & ¿? \\
\hline 494 & Roma-Tirrenos & Dion.Hal.VII.12-13. & ¿? \\
\hline 494 & Roma-Llanura Pomptina & Dion.Hal.VII.1.3-6. & ¿? \\
\hline (volscos) & & Dion.Hal.VII.1.3.-6. & \\
\hline 494 & Roma- Sicilia & Dion.Hal.VII.2.2-5. & \\
\hline 494 & Roma-Cumas & & \\
\hline 491 & Roma-Anciates & Dion.Hal.VII.3.7. & ¿? \\
\hline 489 & Ecuos- Roma & Dion.Hal.VIII.9-10. & ¿? \\
\hline 489 & Roma-Liga Latina. & Dion.Hal.VIII.37;38. & $\begin{array}{l}10 \text { excónsules (I emb), } \\
\text { Aúgures, Pontfices } \\
\text { (II), Mujeres (III y no } \\
\text { autorizada) }\end{array}$ \\
\hline 487 & Roma- Hérnicos & Dion.Hal.VIII.64.1-3. & ¿? \\
\hline 482 & Roma-Veyes & Dion.Hal. VIII.92.1-2. & \\
\hline 481 & Liga Latina-Roma & Dion.Hal.VIII.91.2. & ¿? \\
\hline 478 & Liga Latina-Roma & Dion.Hal.IX.16.2 & ¿? \\
\hline 478 & Veyes-Roma & Dion.Hal.IX.16.2 & ¿? \\
\hline 477 & Veyes-Roma & Dion.Hal.IX,18.3-5. & ¿? \\
\hline 477 & $\begin{array}{l}\text { Veyes-Roma (campamento } \\
\text { military del Cónsul) }\end{array}$ & Dion.Hal.IX.36.2-3. & ¿? \\
\hline 466 & Latinos-Roma & Dion.Hal.IX.66.3-6. & $\begin{array}{l}\text { Q.Fabio y } 2 \text { senadores } \\
\text { adicionales. }\end{array}$ \\
\hline 451 & Roma-Atenas & Dion.Hal.X.52.4. & $\begin{array}{l}\text { Espurio Postumio, } \\
\text { Servio Sulpicio y Aulo } \\
\text { Manlio. }\end{array}$ \\
\hline
\end{tabular}


Notas

1. Eilers,C. et al. Diplomats and Diplomacy in the Ancient World. Leiden, Paises Bajos: Brill, [2009]. p.1.

2. Ibid.

3. Tenemos el mismo caso en Grecia. „The simple unbureaucratic structure of early Greek states did not encourage the provision in advance of institutions directed to the initiation and systematic practice of diplomacy." Adcock, F y Mosley.D.J. Diplomacy in Ancient Greece. London, United Kingdom: Thames and Hudson, [1974]. p.11.

4. Eilers, C. Loc.cit.

5. "Therein lay the origin of diplomatic activity and the need of skilful negotiations to isolate and remove the causes of tension and hostility by persuasion, which is the prime duty of a diplomat" Ibid; p.12.

6. "So far as diplomacy is a skill in that art, it was bound to attract to itself men of experience in affairs and men who possessed a cool appretiation of problems. Such men of natural authority were to be found in the aristocratic councils, which tended to be the place where foreign policy was determined by the Elders, men of admitted wisdom." Ibid.

7. Adcock, F y Mosley, D.J. Op.Cit.

8. IT WAS THE INTENTION of the late Professor Adcock to write a book on Ancient Greek and Roman Diplomacy for this series, and by the time of his death he had assambled notes and typescript in preparation."Ibid; p.7.

9. Jones, Ch. Kinship Diplomacy in the Ancient World. Cambridge Mass., United States: Harvard University Press, [1999].

10. Eilers, C. Op.Cit.

11. Canali De Rossi, F Le ambascerie dal mondo Greco a Roma En: Età reppublicana. Istituto Italiano per la storia antiqua. 1997,63.

12. Cornell, T.J. The Beginnings of Rome. London, United Kingdom: Routledge, [1995].
13. Quae ab condita urbe Roma ad captam eandem urbem Romani sub regibus primum, consulibus deinde ac dictatoribus decemvirisque ac tribunis consularibus decmvirisque ac tribunis consularibus gessere, foris bella, domi seditiones, quinque libris exposui, res cum vetustate nimia obscuras, velut quae magno ex intervallo loci vix cernuntur, tum quod parvae er rarae per eadem tempora litterae fuere, una custodia fideis memoriae rerum gestarum, et quod, etiam si quae in comentariis pontificum aliis que publicis privatisque erant monumentis, incensa urbe peraeque interiere.“ Liv. VI.1.

14. Sobre la Roma primitiva algunas obras de carácter general. Alföldi, A. Early Rome and the Latins. Michigan, United States: Ann Harbor, (1964). Arnold, History of Rome. Early History to the Burning of Rome by the Gauls (2010), Forsythe, A Critical History of Early Rome. From Prehistory to the First Punic War. Berkeley, United States: University of California Press, (2006). Bloch, R The Origins of Rome. London, United Kingdom: Thames and Hudson, (1960), Cornell, The Beginnings of Rome: Italy and Rome from the Bronze Age to the Punic Wars 1000-264 B.C. London, United Kingdom: Routledge (1995),De Francisci, Primordia Civitatis. Roma, Italia: Romae Apollinaris (1959), Gjerstad, E Early Rome i-iv. Lund, Sverige: Svenska Institutet i Rom.(1953-67),J.Heurgon, Rome et la Mediterraneé occidentale jusqu'aux guerres puniques. Paris,France. Presses Universitaires de France.(1969), Holloway, The Archaeology of Early Rome and Latium. Routledge, United Kingdom: Thames and Hudson, (1996), Whatmough, J The Foundations of Roman Italy.London, United Kingdom.Methuen,(1937), Gjerstad, E. Legends and Facts of Early Roman History. Lund, Sverige: Svenska Institutet i Rom (1962), Müller-Karpe, Vom Anfang Roms. Heidelberg, Deutschland: Kerle (1959), Bell, Sinclair y Nagy, H. New Perspectives on Etrury and Early Rome. Wisconsin, United States: University of Wisconsin Press, (2009), Scullard, H.H., The Etruscan Cities and Rome. London, United Kingdom:Thames and Hudson (1967), Gjerstad, E. Les Origines de la Republique Romaine. Entretiens Hardt. 1967. Xiii. Obras de carácter general sobre la historia de Roma: Altheim, Römische Geschichte. Bis Zur Schlacht bei Pydna, 168 v.Chr.Berlin, Deutschland: Walter de Gruyter\& Co.(1956), Bengston, Grundriss der Römischen Geschichte. Munich, Deutschland: Beck (1967), Bleicken, J. Geschichte der Römischen Republik. VI ed. München, Deutschland: Oldenbourg (1988), Carcopino, Les Étapes de l'Imperialisme Romain. Paris, France: Hachette, (1961), Crawford, The Roman Republic. II de. Harvard, United States: Harvard University Press (1993), Crawford, M Early 
Rome and Italy, En: Griffin, J et al. The Oxford History of the Roman World. I ed. Paperback. Oxford, United Kingdom: Oxford University Press(1991), Finley, Politics in the Ancient World. Cambridge, United Kingdom: Cambridge University Press, (1991), Heuß, Römische Geschichte. Braunschweig, Deutschland: Westermann (1960), Kornemann, Römische Geschichte. Die Zeit der Republik. Stuttgart, Deutschland: A.Kröner,(1938), Mommsen, Römische Geschichte. Leipzig,Deutschland: Reimer \& Hirsel(1854), Niebuhr, Vorträge über römische Geschichte. Berlin, Deutschland: G.Reimer Verlag (1828), Pais, Histoire Romaine. Paris, France: A Colin, (1926), Rostovtzeff, M. Rome. II Paperback ed. Oxford, England: Oxford University Press. (1960), Scullard, A History of the Roman World 753 to 146 B.C. V ed. London, United Kingdom: Routledge. [1991].

15. Roma era solamente una de las villas pastoriles de origen latino en el Lacio (un pueblo indoeuropeo que formaba una rama de los indoeuropeos llegados a la península itálica junto a los Umbros y a los Sabélicos).

16. "The expulsion of the Etruscan dynasty, which our tradition refers to the very end of the sixth centry, did not, as I have said already, produce any radical changes in the constitutions of the city. Rome was still a strong, mainly military power, with an aristocracy ruling over a population which was chiefly agricultural.” Rostovtzeff, M. Op.Cit. p.24.

17. Ver Anexo. Mapa 2.

18. The Etruscan cities looked with displeasure on the growing commercial importance which had brought about a treaty of agreement with Carthage. Nearer home her prospects were brighter., for the Latins felt that, without the co-operation of Roman power; it would be difficult for them to either the constant pressure of the Volsci and Aequi from the mountains or the standing danger of a new Etruscan conquest. Hence, in view of their common interests, it was natural that the alliance of all Latin communities should be consolidated at this time; and Rome again began, as in the time of the Etruscan predominance to play a leading part in this federation." Ibid; p.28.

19. "The fall of the monarchy also meant the partial loss of superiority which Rome had achieved visà-vis her immediate neighbours. Furthermore, in the fith century the Volscians emerged from the upper Liris valley and conquered most of the Trera valley and the coastal plain south of Rome. The first century and a half of the Republic saw first the reassertion of Roman leadership of the other Latin communities and then a long sequence of wars against the southern etruscan cities, principally Veii (captured and destroyed in 396), and against the Volscians to the south." Michael Crawford. Early Rome and Italy. III ed. London, United Kingdom: Routledge, [2001]. p.26.

20. Dion.Hal. V.1. 7. Este discurso bien podría haber sido invención de Dionisio de Halicarnaso quien era él mismo un profesor de retórica, autor de tratados sobre el tema y admiraba a los grandes retóricos griegos como Isócrates, Lisias y Demóstenes.

21. Liv. II, 3.5-7. Dion.Hal.V.2.1-5.

22. Liv. II.4.7

23. Liv. II.9.6-7.; Livio informa también que la plebe fue eximida del pago del portazgo y que la venta de sal, antes en manos de particulares, fue transferida al estado y las tasas de guerra serían pagadas por la capa alta de la población. Esto hizo que la plebe no pudiera ser seducida en las penurias del asedio por políticos que pudiesen buscar influenciarla.

24. Dion. Halic.V.4.14

25. Dion. Halic. V.4.1

26. Liv. II.13. 1-5.

27. Dion. Halic.V.6.4.

28. Liv.II.15. 1-2.

29. Dion.Halic.V.9.1

30. Dion.Halic.V.9.2. Roma conquistará definitivamente a los sabinos el 290 a.de J.C.

31. Dion. Halic.V.10.2. La Liga de Ferentina englobaba desde el siglo VI al IV a una federación de ciudades latinas la que es sometida por Roma en 338 a.de J.C.

32. Dion. Halic. V.11.1.

33. Dion. Halic. V.11.4.

34. Dion. Halic. V.11.4. 
35. Dion.Halic. V.11.8.

36. Dion. Halic. V.13.6-7.

37. Dion. Halic. V.13.9.

38. Dion. Halic. V.14.14.

39. Dion. Halic. VI.2.26

40. Dion. Halic. VI. 2.27-28

41. Liv. II.33.4.. Espurio Casio toma la posesión del consulado en el año en que la plebe se escindió en 494 a.de J.C. Casio captura en una campaña existosa contra los volscos, Longula y Polusca y sitia Corioli. También nos informa Dionisio de Halicarnaso que una legación de todas las ciudades latinas opuestas a la guerra llega a Roma: Dion.Halic.VI.2.31.

42. Liv. II.22.2-7.

43. Dion. Halic. VII.1.3-6; 2.1-5.

44. Dion. Halic. VII.2.2-5. Los embajadores lograron escapar ilesos, pero sin nada en las manos. Dion. Halic. VII.12.3.

45. Dion. Halic. VII.12.3.

46. Plut.Coriol. VI.

47. Plut.Coriol.XII

48. Dion.Halic.VII.37.4.

49. Liv. II.30.8.

50. Dion.Halic.VIII.9-10

51. Dion.Halic.VIII.15.2.

52. Dion.Halic. VIII.36. Coriolano era un patricio que desterrado por la plebe y con ánimo de vengarse de ella se alía a los volscos y comanda sus tropas con el propósito de someter su antigua ciudad natal. Tras llevar a cabo un efectivo asedio tres embajadas le son enviadas de las cuáles la última es encabezada por su madre y su esposa. Conmovido Coriolano ordena que se levante el sitio y son los volscos, que en castigo a su nueva traición, lo condenan a muerte. Es imposible, con las fuentes que disponemos, establecer la veracidad de esta historia.

53. Dion.Halic.VIII.38.1.

54. Dion.Halic. VIII.38.3.

55. Dion.Halic. VIII.39-54.Plut.Coriol. XXXI; XXXII;XXXIII. La primer embajada compuesta por diez senadores que nos menciona Dionisio de Halicarnaso es probablemente la misma que nos menciona Plutarco que estaba integrada por familiares de Marco Coriolano.

56. Dion.Halic.VIII.64.1-3. Plut.Coriol. XXXI; XXXII;XXXIII. Los hernicios eran un pueblo que habitaba el valle del Trero, probablemente tenían un dialecto latino pero la forma de su nombre parece osca. Hernici En: Hammond, N.G.L. y Scullar H.H. The Oxford Classical Dictionary. $10^{\text {th }}$ ed. Oxford, United Kingdom:Oxford Clarendon Press, [1991], p.505.

57. Dion.Halic.VIII.91.1-2.

58. Dion.Halic.VIII.9.1-2.

59. Dion.Halic.IX.16.2.

60. Dion.Halic.IX.17.1

61. Dion.Halic.IX.18.3-5.

62. Dion.Halic.IX.36.2-3.

63. Dion.Halic. IX.60.3-6.

64. Liv. III.33. 4-6. La Ley de las XII Tablas es redactada precisamente en este período. Ver también: Diodorus. XII.41.1.

65. Dion.Halic.X.52.4

66. Liv.III.50.16.

67. Liv.IV.9.1-3.

68. Liv.IV.58.1.

69. Plut. Camill. IV. 
70. Plut.Camill.X

71. Liv.V.35.4-5. Véase Dio. VII.25. Plut. Camill.XVII.

72. Liv.V.36.6-8. Dio.VII.25. Zon.VII.2.

73. Liv.V.36.9-11. Dio.VII.25.

\section{Fuentes primarias}

Livy. History of Rome. Vol.I. (Edic.bil. Traducción B.O.Foster). Harvard, United States: Harvard University Press.Loeb Classical Library, [1919]. 484p.

Livy. 1922. History of Rome. Vol.II. (Edic.Bil. Traducción B.O. Foster). Harvard, United States: Harvard University Press.Loeb Classical Library,[1922].480p.

Livy.1924. History of Rome. Vol. III. (Edic. Bil. Traducción B.O. Foster). Harvard, United States: Harvard University Press.Loeb Classical Library, [1924]. 544p.

Titi Livi. Ab Urbe Condita Libri. Pars I. Teubner,[1887].358p.

Denys d' Halicarnasse 1723. Les Antiquités Romaines (Ed. Bil). Paris. [1723] En: [PDF]. http://mercure.fltr.ucl.ac.be/ Hodoi/concordances/denys_hal_ant rom_01/lecture/default.htm [Consulta: 25 de Noviembre-4 de Diciembre de 2010]

Dio Cassius. Vol.VII. Roman History. (Ed.Bil. Traducción E.Cary y H. Foster). Harvard, United States: Harvard University Press. Loeb Classical Library,[1914]. 464p.

Plutarch. Lives IV. (Edic.Bil. Traducción B.Perrin). Harvard, United States: Harvard University Press.Loeb Classical Library, [1916]. 480p.
Plutarch 1914. Lives II. (Edic.Bil. Traducción B.Perrin). Harvard, United States: Harvard University Press.Loeb Classical Library,[1914]. 630p.

\section{Bibliografía}

Adcock, Sir. Diplomacy in Ancient Greece. London, United Kingdom:Thames and Hudson, [1975].287p.

Arnold,T. History of Rome. Early History to the Burning of Rome by the Gauls. New York, United States: D. Appleton \& Co, [1853], 678p.

Alföldi, A. Early Rome and the Latins. Michigan, United States: University of Michigan Press, [1964], 458p.

Bloch, R. The Origins of Rome. London: Thames and Hudson, [1960]. 212p.

Cornell, T. The Beginnings of Rome: Italy and Rome from the Bronze Age to the Punic Wars 1000-264 B.C. London, United Kingdom: Routledge,[1995]. 470p.

Eilers, C. Diplomacy and Diplomats in Ancient Rome. Leiden, Kingdom of the Netherlands: Brill,[2009], 254p.

Forsythe, A 2006. Critical History of Early Rome. From Prehistory to the First Punic War. California, United States: University of California Press, [2006]. 400p.

Heurgon, J. Rome et la Mediterraneé occidentale jusqu'aux guerres puniques. IIIed. Paris, France: Editions Universitaires de France, [1969]. 488p.

Holloway, R. The Archaeology of Early Rome and Latium. IIIed. London, United Kingdom: Routledge, [2000].203p. 
Jones, C.P. Kinship and Diplomacy in the Ancient World. Cambridge Mass., United States: Harvard University Press, [1999].193p.

Müller-Karpe,H. Vom Anfang Roms. Heidelberg, Deutschland: Kerle Verlag. [1959].

Nagy,H et als. New Perspectives on Etrury and Early Rome. Wisconsin, United
States: Wisconsin Studies in Classics. [2009].400 p.

Whatmough, J. The Foundations of Roman Italy. London, United Kingdom: Methuen,[1937].420p.

Scullard, H.H. The Etruscan Cities and Rome. Baltimore, United States. John Hopkins Press. [1988], 384p. 
